\title{
Perspective of Arguments in Public Health
}

\author{
Dinesh Kumar \\ Associate Professor, Community Medicine, Dr. Rajendra Prasad Government Medical College, \\ Kangra at Tanda, Himachal Pradesh, 176001
}

Recently, an argument was put forth because a symptomatic and positive patient for CoVID-19 turned tested negative after 7 days, so discharged from the hospital. Both at the time of admission and discharge real-time reverse transcriptase Polymerase Chain Reaction (RTPCR) was done for testing of CoVID-19. Immediately, patient again developed respiratory symptoms and was admitted to hospital again. Amidst of current CoVID-19 pandemic, a question was asked "What is the specificity of the Real Time-Polymerase Chain Reaction (RT-PCR) test for COVID-19?" with an assumption that what if at the time of discharge the disease is present in patient but test turned out to be negative? In response to that a counter statement was posed that "It is the sensitivity that should be asked rather than specificity". It was based on the implication of primary question that was implying false negative report of the RT-
PCR. It means, since patient was discharged with negative result that could be false negative.

The counter statement raised a fundamental concern to primary question so it was asked to be explained. Discussion began with a hypothetical scenario, wherein it was assumed that in a pool of 1,000 persons 500 are positive for disease (CoVID-19) and 500 are negative. For RT-PCR, sensitivity of $50.0 \%$ and specificity of $50.0 \%$ were considered for illustration purpose. It was also assumed that there is no change in method of sampling and testing. This scenario resulted 250 number for each of true positives (TPs), false positives (FPs), true negatives (TNs) and false negatives (FNs). (Table 1) Recall primary argument where patient was negative at the time of discharge and question was asked about specificity of RT-PCR and counter statement referred to sensitivity rather than specificity.

TABLE 1 EXPECTED NUMBERS OF POSITIVES AND NEGATIVES AMONG HYPOTHETICAL 500 DISEASED AND 500 NON-DISEASED USING RT-PCR ASSUMING 50.0\% SENSITIVITY AND 50.0\% SPECIFICITY.

\begin{tabular}{|c|c|c|c|c|}
\hline & & \multicolumn{2}{|c|}{ COVID-19 } & \\
\hline & & Positive & Negative & \\
\hline \multirow[t]{3}{*}{ RT-PCR } & Positive & $\begin{array}{c}500 \times 0.50=250 \\
\text { (True Positives) }\end{array}$ & $\begin{array}{l}500-250=250 \\
\text { (False Positives) }\end{array}$ & 500 \\
\hline & Negative & $\begin{array}{c}500-250=250 \\
\text { (False Negatives) }\end{array}$ & $\begin{array}{l}500 \times 0.50=250 \\
\text { (True Negatives) }\end{array}$ & 500 \\
\hline & & 500 & 500 & 1000 \\
\hline
\end{tabular}

Let's discuss counter statement first, mentioning implication of sensitivity to negative test result at the time of discharge. Sensitivity hints about number of test-based diseased among actual diseased that is TPs. Since primary question asked about specificity i.e., TNs which intuitively implies false negativity of the test or what if, the test is false negative? It is correct, that FNs contribute to denominator of sensitivity rather than specificity and counter statement implies

CORRESPONDING AUTHOR: Dr. Dinesh Kumar, Associate Professor, Community Medicine, Dr. Rajendra Prasad Government Medical College, Kangra at Tanda, Himachal Pradesh, 176001.

E Mail ID: dinesh9809@gmail.com

ARTICLE CYCLE: Received: 02/12/2021; Revised: 24/03/2021; Accepted: 27/05/2021; Published:30/06/2021

CITATION: Kumar D. Perspective of Arguments in Public Health. J Comp Health. 2021;9(1):44-45. 
that patient turned out negative at the time of discharge could be false negative.

So, primary question stands correct or incorrect? Further discussion to primary question deliberates that negative test result implies absence of disease. Reading the primary question in the light that what if test has high specificity? let's say $100.0 \%$ with fixed sensitivity of $50.0 \%$ then there is no reason believe that disease might not be absent in patient at the time of discharge. It can also be reiterated that probability of false negative test decreases from $50.0 \%(250 / 500 \times 100)$ to $33.3 \%(250 / 750 \times 100)$ and probability of presence of disease and false positivity are is absent. (Table 2 ) The probability of FNs also called as negative predictive value of (NPV) of test.

TABLE 2 EXPECTED NUMBERS OF POSITIVES AND NEGATIVES AMONG HYPOTHETICAL 500 DISEASED AND 500 NON-DISEASED USING RT-PCR ASSUMING 50.0\% SENSITIVITY AND 100.0\% SPECIFICITY.

\begin{tabular}{|l|l|c|c|c|}
\hline & & \multicolumn{4}{|c|}{ COVID-19 } & Negative \\
\hline \multirow{2}{*}{ RT-PCR } & Positive & Positive & $500-500=0$ & 250 \\
& & $500 \times 0.50=250$ & (False Positives) & \\
\cline { 2 - 5 } & Negative & $500-250=250$ & $500 \times 1.0=500$ & 750 \\
& & (False Negatives) & (True Negatives) & 1000 \\
\hline
\end{tabular}

Going back to primary question again, if negative test result at the time of discharge is not to be believed. It left with a question that where that patient could be in the confusion matrix? In reality, patient actually is disease negative but low specificity increases the probability of patient in FPs cell i.e., patient could have tested false positive. If in reality, patient is positive with disease so test with low specificity increases the probability of patient either in TPs or FNs cell i.e., patient has disease at the time of discharge and could have tested either positive or negative.

Sensitivity and specificity are inversely related with each other. It is the sensitivity which tells about positives in disease or probability of being positive test when disease is present. Whereas, specificity measures negative in health or probability of negative test when disease is absent. Understanding further, if a highly specific test is positive it rules the presence of disease. It means that if test turned out positive the probability of presence of disease is highest. On the contrary, if a highly sensitive test is negative it rules out the presence of disease. It implies that in a case of negative test report the probability of absence of disease highest. ${ }^{[1]}$ In this discussion, we have not assumed a change in the cut-off criteria.
Following this rule where the primary question asked about the specificity of test stands valid as negative report of a highly specific test favour against the presence of disease that means probability of absence of disease is highest. Let's discuss if answer to the primary question reveals that the test has poor specificity than assumption will be that the negative test report poorly favour against presence of disease. That means the probability of presence of disease increases, so as the chances of high test sensitivity as it relates inversely with specificity.

In truth, the actual disease status remains unknown and it is right that, if we assume that the test result could be false negative it is sensitivity or 1-senstivity should be asked rather than specificity. But in a case if the test has highest of specificity i.e., $100.0 \%$ then we are certain that disease might be truly absent at the time of discharge. As a result, it is the implication of question which decides the course of argument to follow. In current situation primary question asked about probability of absence of disease whereas counter statement implied about validity of test.

\section{REFERENCES}

1. Parikh R, Mathai A, Parikh S, Chandra Sekhar G, Thomas R. Understanding and using sensitivity, specificity and predictive values. Indian J Ophthalmol. 2008;56(1):45-50. doi:10.4103/0301-4738.37595 